\title{
ON THE WEIGHTED APPROXIMATION OF CONTINUOUSLY DIFFERENTIABLE FUNCTIONS
}

\author{
LEOPOLDO NACHBIN
}

(Communicated by Palle E. T. Jorgensen)

\begin{abstract}
This note is an improvement of the available methods for getting results on the weighted approximation of continuously differentiable functions.
\end{abstract}

We shall present what we believe to be a simplified version of the reasoning to establish some results concerning weighted approximation of continuously differentiable scalar functions on $\mathbf{R}^{n}$ (see [3]). For references to weighted approximation of continuous scalar functions on $\mathbf{R}^{n}$ (see [1] and [3]). Lemma 1 reduces the search for sufficient conditions in order that a weight on $\mathbf{R}$ be $C^{m}$-fundamental to the finding of sufficient conditions for a weight on $\mathbf{R}$ to be $C$-fundamental. Similarly, Lemma 2 reduces the finding of sufficient conditions for a weight on $\mathbf{R}^{n}$ to be $C^{m}$-fundamental to the search of sufficient conditions for a weight on $\mathbf{R}$ to be $C^{m}$-fundamental. We then apply Lemmas 1 and 2 together to obtain Propositions 2, 4, and 5.

Fix integers $n \in \mathbf{N}, n \geq 1$, and $m \in \mathbf{N}$ (the case $m=\infty$ will be excluded since it follows easily from all $m \in \mathbf{N}$ ). Let $\mathbf{K}$ denote either $\mathbf{R}$ or $\mathbf{C}$. Consider the algebras $\mathscr{P}\left(\mathbf{R}^{n}\right)$ of all $\mathbf{K}$-valued polynomials on $\mathbf{R}^{n}$ and $C^{m}\left(\mathbf{R}^{n}\right)$ of all continuously $m$-differentiable K-valued functions on $\mathbf{R}^{n}$. Write $\mathbf{N}_{m}^{n}=\{\alpha \in$ $\left.\mathbf{N}^{n}:|\alpha| \leq m\right\}$ where $|a|=\alpha_{1}+\cdots+\alpha_{n}$ if $\alpha=\left(\alpha_{1}, \ldots, \alpha_{n}\right) \in \mathbf{N}^{n}$. Let $D^{\alpha} f$ be the $\alpha$ th partial derivative of $f \in C^{m}\left(\mathbf{R}^{n}\right)$ for $\alpha \in \mathbf{N}_{m}^{n}$. Denote by $\mathscr{D}^{m}\left(\mathbf{R}^{n}\right)$ the subalgebra of $C^{m}\left(\mathbf{R}^{n}\right)$ of all functions with compact support.

A $C^{m}$-weight on $\mathbf{R}^{n}$ is a family $v=\left\{v_{\alpha}: \alpha \in \mathbf{N}_{m}^{n}\right\}$ of upper semicontinuous functions $v_{\alpha} \geq 0$ on $\mathbf{R}^{n}$. Such a weight $v$ defines the vector space $C^{m} v_{\infty}\left(\mathbf{R}^{n}\right)$ of all $f \in C^{m}\left(\mathbf{R}^{n}\right)$ such that $v_{\alpha} D^{\alpha} f$ tends to zero at infinity for every $\alpha \in \mathbf{N}_{m}^{n}$. Set $\|f\|_{v_{\alpha}}=\sup \left\{v_{\alpha}(t) \cdot\left|D^{\alpha} f(t)\right|: t \in \mathbf{R}^{n}\right\}$ to get a seminorm $f \in C^{m} v_{\infty}\left(\mathbf{R}^{n}\right) \mapsto\|f\|_{v} \in \mathbf{R}_{+}$for every $\alpha \in \mathbf{N}_{m}^{n}$. The finite family of such seminorms makes $C^{m^{\alpha}} v_{\infty}\left(\mathbf{R}^{n}\right)$ into a seminormable space, actually a

Received by the editors November 21, 1989.

1980 Mathematics Subject Classification (1985 Revision). Primary 46E15.

Key words and phrases. Weighted approximation, fundamental weight, analytic weight, quasianalytic weight. 
seminormed space if for instance we use the seminorm $f \in C^{m} v_{\infty}\left(\mathbf{R}^{n}\right) \mapsto$ $\|f\|_{v}=\sup \left\{\|f\|_{v_{\alpha}}: \alpha \in \mathbf{N}_{m}^{n}\right\}=\sup \left\{v_{\alpha}(t) \cdot\left|D^{\alpha} f(t)\right|: t \in \mathbf{R}^{n}, \alpha \in \mathbf{N}_{m}^{n}\right\} \in \mathbf{R}_{+}$. We now have that $\mathscr{D}^{m}\left(\mathbf{R}^{n}\right) \subset C^{m} v_{\infty}\left(\mathbf{R}^{n}\right)$. It is known that $\mathscr{D}^{m}\left(\mathbf{R}^{n}\right)$ is dense in $C^{m} v_{\infty}\left(\mathbf{R}^{n}\right)$ if the weight $v$ is decreasing in the sense that, for every $\alpha$, $\beta \in \mathbf{N}_{m}^{n}$ with $\beta \leq \alpha$, there exists $C_{\alpha \beta} \geq 0$ such that $v_{\alpha} \leq C_{\alpha \beta} v_{\beta}$ ([3, Lemma 1]). $\mathscr{D}^{m}\left(\mathbf{R}^{n}\right)$ may be dense in $C^{m} v_{\infty}\left(\mathbf{R}^{n}\right)$ even if $v$ is not decreasing, and it may fail to be dense as well. The weight $v$ is said to be rapidly decreasing if $\mathscr{P}\left(\mathbf{R}^{n}\right) \subset C^{m} v_{\infty}\left(\mathbf{R}^{n}\right)$. If, moreover, $\mathscr{P}\left(\mathbf{R}^{n}\right)$ is dense in $C^{m} v_{\infty}\left(\mathbf{R}^{n}\right)$, then $v$ is called $C^{m}$-fundamental.

Lemma 1. Let $m \in \mathbf{N}, v_{i} \geq 0, i=0, \ldots, m$, and $u \geq 0$ be upper semicontinuous on $\mathbf{R}$. Consider the $C^{m}$-weight $v=\left(v_{0}, \ldots, v_{m}\right)$ on $\mathbf{R}$ which is assumed to be decreasing. Let $u$ be $C$-fundamental on $\mathbf{R}$ such that $s, t \in \mathbf{R}$, $|s| \leq|t|$ imply $u(t) \leq u(s)$ and $|t|^{m-i} v_{i}(t) \leq u(t), v_{i}(t) \leq u(t)$ for $t \in \mathbf{R}$, $i=0, \ldots, m$. Then $v$ is $a C^{m}$-fundamental weight on $\mathbf{R}$.

Proof. The lemma is true if $m=0$ because then $v_{0} \leq u$ and hence $v_{0}$ is $C$-fundamental along with $u$. Assume now that $m \geq 1$ and that the lemma is true for $m-1$. Notice that $\mathscr{P}(\mathbf{R}) \subset C^{m} v_{\infty}(\mathbf{R})$ because $\mathscr{P}(\mathbf{R}) \subset C u_{\infty}(\mathbf{R})$ and $v_{i} \leq u, i=0, \ldots, m$. Hence $v$ is rapidly decreasing. Fix any $f \in \mathscr{D}^{m}(\mathbf{R})$ and $\varepsilon>0$. Then $f^{(m)} \in \mathscr{D}(\mathbf{R}) \subset C u_{\infty}(\mathbf{R})$ and there is a $P^{(m)} \in \mathscr{P}(\mathbf{R})$ so that

$$
u(t) \cdot\left|P^{(m)}(t)-f^{(m)}(t)\right| \leq \varepsilon, \quad t \in \mathbf{R} .
$$

Choose $P \in \mathscr{P}(\mathbf{R})$ whose $m$ th derivative is $P^{(m)}$ such that $P^{(i)}(0)=f^{(i)}(0)$, $i=0, \ldots, m-1$. We claim that

$$
v_{i}(t) \cdot\left|P^{(i)}(t)-f^{(i)}(t)\right| \leq \varepsilon, \quad t \in \mathbf{R}, i=0, \ldots, m .
$$

In fact, for $i=m$ this follows from (1) and $v_{m} \leq u$. For every $t \in \mathbf{R}$, $i=0, \ldots, m-1$, there is an $s \in \mathbf{R},|s| \leq|t|$ such that $\left|P^{(i)}(t)-f^{(i)}(t)\right| \leq$ $|t|^{m-i} \cdot\left|P^{(m)}(s)-f^{(m)}(s)\right|$ by the mean value theorem applied $m-i$ times. Notice that $|t|^{m-i} v_{i}(t) \leq u(t) \leq u(s)$. Hence (1) with $s$ in place of $t$ shows that (2) is true for $i=0, \ldots, m-1$ as well as for $i=m$. It follows that $\mathscr{D}^{m}(\mathbf{R})$ is contained in the closure of $\mathscr{P}(\mathbf{R})$ in $C^{m} v_{\infty}(\mathbf{R})$. Therefore, $\mathscr{P}(\mathbf{R})$ is dense in $C^{m} v_{\infty}(\mathbf{R})$ along with $\mathscr{D}^{m}(\mathbf{R})$. Thus $v$ is $C^{m}$-fundamental.

Let $v \geq 0$ be upper semicontinuous on $\mathbf{R}$. We say that $v$ is an analytic weight on $\mathbf{R}$ when there exist $C>0, c>0$ such that $v(t) \leq C e^{-c|t|}, t \in \mathbf{R}$. It is then known that $v$ is a $C$-fundamental weight on $\mathbf{R}$ ([2, §28, Lemma 2]). More generally, if $v \geq 0$ is upper semicontinuous on $\mathbf{R}^{n}$, we say that $v$ is an analytic weight on $\mathbf{R}^{n}$ when there exist $C>0, c>0$ such that $v(t) \leq$ $C e^{\left.-c\left(\left|t_{1}\right|\right)+\cdots+\left|t_{n}\right|\right)}, t \in \mathbf{R}^{n}$. It is then known that $v$ is $C$-fundamental on $\mathbf{R}^{n}$.

Proposition 2. Let $m \in \mathbf{N}, v_{i} \geq 0, i=0, \ldots, m$, be upper semicontinuous on $\mathbf{R}$. Consider the $C^{m}$-weight $v=\left(v_{0}, \ldots, v_{m}\right)$ on $\mathbf{R}$ which is assumed to be decreasing. Let each $v_{i}, i=0, \ldots, m$, be an analytic weight. Then $v$ is a $C^{m}$-fundamental weight on $\mathbf{R}$. 
Proof. Assume that $v_{i}(t) \leq C e^{-c|t|}, t \in \mathbf{R}, i=0, \ldots, m$, for some $C>0$, $c>0$. Choose $D>0,0<d<c$ so that, if $u(t)=D e^{-d|t|}, t \in \mathbf{R}$, all assumptions in Lemma 1 are satisfied.

Lemma 3. Let $n \in \mathbf{N}, n \geq 1, m \in \mathbf{N}, v_{\alpha} \geq 0, \alpha \in \mathbf{N}_{m}^{n}$, be upper semicontinuous on $\mathbf{R}$. Consider the $C^{m}$-weight $v=\left(v_{\alpha} ; \alpha \in \mathbf{N}_{m}^{n}\right)$ on $\mathbf{R}^{n}$ which is assumed to be decreasing. Let $u_{i j} \geq 0, i=1, \ldots, n, j=0, \ldots, m$, be upper semicontinuous on $\mathbf{R}$. Consider the $C^{m}$-weights $u_{i}=\left(u_{i 0}, \ldots, u_{i m}\right), i=1, \ldots, n$, on $\mathbf{R}$ which are supposed to be decreasing and $C^{m}$-fundamental. Assume

$$
v_{\alpha}(t) \leq u_{1 \alpha_{1}}\left(t_{1}\right) \ldots u_{n \alpha_{n}}\left(t_{n}\right), \quad t \in \mathbf{R}^{n}, \alpha \in \mathbf{N}_{m}^{n} .
$$

Then $v$ is $C^{m}$-fundamental on $\mathbf{R}^{n}$.

Proof. Consider the $n$-linear mapping $\pi$ that, with every

$$
\left(f_{1}, \ldots, f_{n}\right) \in C^{m}\left(u_{1}\right)_{\infty}(\mathbf{R}) \times \cdots \times C^{m}\left(u_{n}\right)_{\infty}(\mathbf{R})
$$

associates $f_{1} \otimes \cdots \otimes f_{n} \in C^{m} v_{\infty}\left(\mathbf{R}^{n}\right)$ where $\left(f_{1} \otimes \cdots \otimes f_{n}\right)(t)=f_{1}\left(t_{1}\right) \cdots f_{n}\left(f_{n}\right)$ for $t \in \mathbf{R}^{n}$. The assumptions make it sure that $\pi$ is well defined and continuous because

$$
\left\|f_{1} \otimes \cdots \otimes f_{n}\right\|_{v_{\alpha}} \leq\left\|f_{1}\right\|_{u_{1 \alpha_{1}}} \ldots\left\|f_{n}\right\|_{u_{n \alpha_{n}}}
$$

By hypothesis, $\mathscr{P}(\mathbf{R})$ is dense in $C^{m}\left(u_{i}\right)_{\infty}(\mathbf{R})$ so $\mathscr{D}^{m}(\mathbf{R})$ is contained in the closure of $\mathscr{P}(\mathbf{R})$ in $C^{m}\left(u_{i}\right)_{\infty}(\mathbf{R}), i=1, \ldots, n$. Thus $\pi\left[\mathscr{D}^{m}(\mathbf{R}) \times \cdots \times \mathscr{D}^{m}(\mathbf{R})\right]$ is contained in the closure of $\pi[\mathscr{P}(\mathbf{R}) \times \cdots \times \mathscr{P}(\mathbf{R})]$ in $C^{m} v_{\infty}\left(\mathbf{R}^{n}\right)$. Hence the vector subspace $\mathscr{D}^{m}(\mathbf{R}) \otimes \cdots \otimes \mathscr{D}^{m}(\mathbf{R})$ generated by $\pi\left[\mathscr{D}^{m}(\mathbf{R}) \times \cdots \times \mathscr{D}^{m}(\mathbf{R})\right]$ is contained in the closure in $C^{m} v_{\infty}\left(\mathbf{R}^{n}\right)$ of the vector subspace $\mathscr{P}\left(\mathbf{R}^{n}\right)=\mathscr{P}(\mathbf{R}) \otimes$ $\cdots \otimes \mathscr{P}(\mathbf{R})$ generated by $\pi[\mathscr{P}(\mathbf{R}) \times \cdots \times \mathscr{P}(\mathbf{R})]$. It is known that $\mathscr{D}^{m}(\mathbf{R}) \otimes \cdots \otimes$ $\mathscr{D}^{m}(\mathbf{R})$ is dense in $\mathscr{D}^{m}\left(\mathbf{R}^{n}\right)$ in the natural inductive limit topology of $\mathscr{D}^{m}\left(\mathbf{R}^{n}\right)$, hence in the coarser topology on $\mathscr{D}^{m}\left(\mathbf{R}^{n}\right)$ defined by the norm $f \in \mathscr{D}^{m}\left(\mathbf{R}^{n}\right) \mapsto$ $\sup \left\{\left|D^{\alpha} f(t)\right|: t \in \mathbf{R}^{n},|\alpha| \leq m\right\} \in \mathbf{R}_{+}$, hence (because all $v_{\alpha}$ are upper bounded along with all $u_{i j}$ ) in the even coarser topology that the natural topology of $C^{m} v_{\infty}\left(\mathbf{R}^{n}\right)$ induces on $\mathscr{D}^{m}\left(\mathbf{R}^{n}\right)$. Since $\mathscr{D}^{m}\left(\mathbf{R}^{n}\right)$ is dense in $C^{m} v_{\infty}\left(\mathbf{R}^{n}\right)$, then $\mathscr{D}^{m}(\mathbf{R}) \otimes \cdots \otimes \mathscr{D}^{m}(\mathbf{R})$ is dense in $C^{m} v_{\infty}\left(\mathbf{R}^{n}\right)$. The fact that $\mathscr{D}^{m}(\mathbf{R}) \otimes \cdots \otimes$ $\mathscr{D}^{m}(\mathbf{R})$ is contained in the closure of $\mathscr{P}\left(\mathbf{R}^{n}\right)$ in $C^{m} v_{\infty}\left(\mathbf{R}^{n}\right)$ implies then that $\mathscr{P}\left(\mathbf{R}^{n}\right)$ is dense in $C^{m} v_{\infty}\left(\mathbf{R}^{n}\right)$. Thus $v$ is $C^{m}$-fundamental.

Proposition 4. Let $n \in \mathbf{N}, n \geq 1, m \in \mathbf{N}, v_{\alpha} \geq 0, \alpha \in \mathbf{N}_{m}^{n}$, be upper semicontinuous on $\mathbf{R}^{n}$. Consider the $C^{m}$-weight $v=\left(v_{\alpha} ; \alpha \in \mathbf{N}_{m}^{n}\right)$ on $\mathbf{R}^{n}$ which is assumed to be decreasing. Assume that each $v_{\alpha}, \alpha \in \mathbf{N}_{m}^{n}$, is an analytic weight. Then $v$ is $C^{m}$-fundamental on $\mathbf{R}^{n}$.

Proof. Let $v_{\alpha}(t) \leq C e^{-c\left(\left|t_{1}\right|+\cdots+\left|t_{n}\right|\right)}, t \in \mathbf{R}^{n}, \alpha \in \mathbf{N}_{m}^{n}$, for suitable $C>0$, $c>0$. Choose $u_{i j}\left(t_{i}\right)=C^{1 / n} e^{-c\left|t_{i}\right|}, i=1, \ldots, n, j=1, \ldots, m$, so that all assumptions in Lemma 3 are satisfied. 
Let $v \geq 0$ be upper semicontinuous on $\mathbf{R}$ and rapidly decreasing. Put $M_{m}=\sup \left\{\left|t^{m}\right| \cdot v(t): t \in \mathbf{R}\right\} \in \mathbf{R}_{+}, m=0,1, \ldots$. We say that $v$ is a quasianalytic weight on $\mathbf{R}$ when $\sum_{m=1}^{\infty}\left(1 / \sqrt[m]{M_{m}}\right)=+\infty$. It is then known that $v$ is a $C$-fundamental weight on $\mathbf{R}$ and that every analytic weight on $\mathbf{R}$ is quasi-analytic (see [2, §29, Lemma 2]). More generally, if $v \geq 0$ is upper semicontinuous on $\mathbf{R}^{n}$, we say that $v$ is a quasi-analytic weight on $\mathbf{R}^{n}$ if there are quasi-analytic weights $v_{1}, \ldots, v_{n}$ on $\mathbf{R}$ such that $v(t) \leq v_{1}\left(t_{1}\right) \cdots v_{n}\left(t_{n}\right)$, $t \in \mathbf{R}^{n}$. It is then known that $v$ is $C$-fundamental on $\mathbf{R}^{n}$, and that every analytic weight on $\mathbf{R}^{n}$ is quasi-analytic.

Proposition 5. Let $n \in \mathbf{N}, n \geq 1, m \in \mathbf{N}, v_{\alpha} \geq 0, \alpha \in \mathbf{N}_{m}^{n}$, be upper semicontinuous on $\mathbf{R}^{n}$. Consider the $C^{m}$-weight $v=\left(v_{\alpha} ; \alpha \in \mathbf{N}_{m}^{n}\right)$ which is supposed to be decreasing. Assume that there are quasi-analytic weights $v_{i}$, $i=1, \ldots, n$, on $\mathbf{R}$ such that $v_{\alpha}(t) \leq v_{1}\left(t_{1}\right) \cdots v_{n}\left(t_{n}\right), t \in \mathbf{R}^{n}, \alpha \in \mathbf{N}_{m}^{n}$. Then $v$ is a $C^{m}$-fundamental weight on $\mathbf{R}^{m}$.

Proof. All assumptions of Lemma 3 apply.

Lemma 6. The linear mapping $D: f \in \mathscr{D}^{1}(\mathbf{R}) \mapsto f^{\prime} \in \mathscr{K}(\mathbf{R})=\mathscr{D}^{0}(\mathbf{R})$ is injective. Its image is $D \mathscr{D}^{1}(\mathbf{R})=\left\{g \in \mathscr{K}(\mathbf{R}) ; \int g=0\right\}$. If $u \geq 0$ is upper semicontinuous on $\mathbf{R}$, this image is dense in $C u_{\infty}(\mathbf{R})$ if and only if $\int 1 / u=$ $+\infty$.

Proof. Only the final part of the lemma requires a proof. Consider the linear form $I: f \in \mathscr{K}(\mathbf{R}) \mapsto \int f \in \mathbf{K}$. Assume $\int 1 / u=+\infty$. We claim that $I$ is not continuous on $\mathscr{K}(\mathbf{R})$ for the seminorm induced by $C u_{\infty}(\mathbf{R})$. In fact, given any $c \geq 0$, there is an $f \in \mathscr{K}(\mathbf{R})$ such that $0 \leq f \leq 1 / u, \int f \geq c$. Therefore $\|f\|_{u} \leq 1$ and $I(f) \geq c$ show that $I$ is not continuous on $\mathscr{K}(\mathbf{R})$ for the seminorm induced by $C u_{\infty}(\mathbf{R})$. It follows that $I^{-1}(0)=D \mathscr{D}^{1}(\mathbf{R})$ is dense in $C u_{\infty}(\mathbf{R})$. Conversely let $c=\int 1 / u<+\infty$. Then the set where $u$ vanishes has a void interior. It follows that the seminorm on $C u_{\infty}(\mathbf{R})$ is actually a norm. We claim that $|I(f)| \leq c \cdot\|f\|_{u}$ for $f \in \mathscr{K}(\mathbf{R})$. This is clear if $\|f\|_{u}=0$. If $\|f\|_{u}>0$ then $u(t)|f(t)| \leq\|f\|_{u}$ implies $|f(t)| \leq\|f\|_{u} / u(t)$ for $t \in \mathbf{R}$, hence $|I(f)| \leq c\|f\|_{u}$ for $f \in \mathscr{K}(\mathbf{R})$ as asserted. Thus $I$ is continuous on $\mathscr{K}(\mathbf{R})$ for the norm induced by $C u_{\infty}(\mathbf{R})$ and $I$ extends uniquely to a continuous linear form $I$ on $C u_{\infty}(\mathbf{R})$, since $\mathscr{K}(\mathbf{R})$ is dense in $C u_{\infty}(\mathbf{R})$. We know that $I \neq 0$ because $I$ does not vanish on $\mathscr{K}(\mathbf{R})$, but $I$ does vanish on $D \mathscr{D}^{1}(\mathbf{R})$. It follows that $D \mathscr{D}^{1}(\mathbf{R})$ is not dense in $C u_{\infty}(\mathbf{R})$.

Example 7. Consider $v_{0}=0, v_{1} \geq 0$ upper semicontinuous on $\mathbf{R}$, and the $C^{1}$ weight $v=\left(v_{0}, v_{1}\right)$ on $\mathbf{R}$. (Notice that $v$ is not decreasing unless $v_{1}=0$.) Then $\mathscr{D}^{1}(\mathbf{R})$ is dense in $C^{1} v_{\infty}(\mathbf{R})$ if and only if $\int 1 / v_{1}=+\infty$.

Proof. Since $v_{0}=0$ we see that $\mathscr{D}^{1}(\mathbf{R})$ is dense in $C^{1} v_{\infty}(\mathbf{R})$ if and only if $D \mathscr{D}^{1}(\mathbf{R})$ is dense in $C\left(v_{1}\right)_{\infty}(\mathbf{R})$. It remains to apply Lemma 6 with $v_{1}$ in place of $u$. 


\section{REFERENCES}

1. J. Horvath, Approximacion y funciones casi-analiticas, Universidad de Madrid, Spain, 1956.

2. L. Nachbin, Elements of approximation theory, Van Nostrand, USA, 1987.

3. G. Zapata, Bernstein approximation problem for differentiable functions and quasi-analytic weights, Trans. Amer. Math. Soc. 182 (1973), 503-509.

Department of MAthematics, University of Rochester, Rochester, New YoRK 14627

Current address: Centro Brasileiro de Pesquisas Fisicas, Rua Xavier Sigaud 150, 22290 Rio de Janeiro, RJ, Brasil 